\title{
Strategies for the Use of Mathematics Textbooks for Primary and Secondary Schools under the Background of "Internet+"
}

\author{
Zongzhao Mo, Ying Zhou* \\ School of Mathematics and Statistics, Guangxi Normal University, Guilin, China \\ Email: ${ }^{* 799936971 @ q q . c o m ~}$
}

How to cite this paper: Mo, Z.Z. and Zhou, Y. (2019) Strategies for the Use of Mathematics Textbooks for Primary and Secondary Schools under the Background of "Internet+". Open Journal of Social Sciences, 7, 197-204.

https://doi.org/10.4236/jss.2019.77018

Received: June 7, 2019

Accepted: July 21, 2019

Published: July 24, 2019

Copyright $\odot 2019$ by author(s) and Scientific Research Publishing Inc. This work is licensed under the Creative Commons Attribution International License (CC BY 4.0).

http://creativecommons.org/licenses/by/4.0/

\begin{abstract}
Mathematics textbooks for primary and secondary schools play an important role in elementary education and the realization of their functions and value depends on the scientific and effective use of teachers and students. Therefore, some strategies are put forward: understand the intentions of the mathematics textbook, explore the educational and teaching value of mathematics textbooks, grasp mathematics textbooks as a whole, use mathematics textbooks creatively, attach importance to the comparative study and use of different versions of mathematics textbooks.
\end{abstract}

\section{Keywords}

Internet+, Primary and Secondary Schools, Mathematics Textbooks, Use Strategies

\section{Introduction}

"Internet+" is a whole process of Internet based integration of information technology, including mobile Internet, cloud computing, artificial intelligence, big data technology, and so on, and has constantly created new products, new businesses and new models in various sectors of the economic and social life [1]. The deep integration of the Internet and education breaks the barriers of time and space, and makes education turn from closed to open. High-quality educational resources are greatly enriched. With the help of the Internet, they flow across regions and schools, realizing "Everyone can learn everywhere and anytime". However, "Internet+ education" has also brought new challenges. A 
prominent problem is that the mathematics textbooks for primary and secondary schools have been marginalized. Mathematics teachers' use of mathematics textbooks is gradually decreasing [2]. In view of this, this paper discusses how to use mathematics textbooks scientifically and effectively under the background of "Internet + " in order to better play the educational functions and value of mathematics textbooks and improve the quality of elementary education.

\section{Strategies for the Use of Mathematics Textbooks for Primary and Secondary Schools}

The special status of mathematics textbooks for elementary and middle schools determines the importance of using mathematics textbooks scientifically and effectively. However, through front-line teaching research and literature review, it is found that due to the influence of the Internet and other factors, the mathematics textbooks of primary and secondary school have not been paid enough attention and the use level of mathematics textbooks is not high. Therefore, it is very necessary for every mathematics teachers to explore scientific and effective strategies for the use of mathematics textbooks in primary and secondary schools under the background of "Internet+".

\subsection{Understanding the Intentions of the Mathematics Textbooks}

A thorough understanding of textbooks is essentially a thorough understanding of the thoughts and intentions of textbook writers [3]. Each example and exercise of the mathematics textbook are selected after many experts and scholars have repeatedly discussed, with profound connotation and rich nutrition. Mathematics teachers can better understand mathematics textbooks, and thus make good use of mathematics textbooks by understanding the writing intentions of mathematics textbooks. For example, why are mathematics textbooks arranged in such a way, why is the content presented in such a way, and what are the purpose and significance of setting expanding knowledge columns such as "mathematics in life". For example, the knowledge presentation method of each section of the High School Mathematics Textbooks of Beijing Normal University Press is "Problem Raising, Analysis and Understanding, Case Analysis, Thinking and Exchanging, Hands-on Practice, Abstract Generalization, Examples, Exercises, Small Materials", and its purpose is to cultivate students' awareness of problems and develop students' ability of independent thinking and independent inquiry, let students experience the occurrence and development of mathematical knowledge, thus understand the role of mathematical knowledge in the development of science and society.

\subsection{Deeply Exploring the Educational and Teaching Value of Mathematics Textbooks}

Mathematics textbooks for primary and secondary schools are different from general reading materials in that they embody the fruits of labor and wisdom of 
numerous mathematics education experts, mathematics scholars and outstanding front-line mathematics teachers. They are not only the carrier of mathematics knowledge, but also the carrier of curriculum standard, spirit, culture, thought and history of mathematics. Therefore, mathematics teachers should read the mathematics textbooks carefully, and fully explore the educational value such as the value of moral education, the value of culture education, the value of science education and the value of innovation education contained in the mathematics textbooks.

\subsubsection{Deeply Excavating the Educational and Teaching Value of the Examples of Mathematics Textbooks}

Example is an important part of the mathematics textbooks with the characteristics of typicality, foundation and exemplary, which plays an important role in consolidating the new mathematics knowledge, revealing mathematics thoughts and methods and so on [4]. Therefore, the mathematics teachers' use of examples cannot be confined to "give students to understand", but should pay attention to the mathematics thoughts and methods, problem solving strategies that contained in examples so as to improve the teaching efficiency and quality of education.

For example, the straight line $l: 3 x+y-6=0$ and A circle with center C: $x^{2}+y^{2}-2 y-4=0$, judge the position relationship between the straight line $l$ and the circle $C$.

Solution method 1: from the equations of the straight line $l$ and the circle $C$, we get:

$$
\left\{\begin{array}{l}
3 x+y-6=0 \\
x^{2}+y^{2}-2 y-4=0
\end{array}\right.
$$

Eliminating $y$, we get $x^{2}-3 x+2=0$, because $\Delta=(-3)^{2}-4 \times 1 \times 2=1>0$, so the straight line $l$ intersects the circle $C$ and has two intersection points.

Solution method 2: $x^{2}+y^{2}-2 y-4=0$ is simplified to $x^{2}+(y-1)^{2}=5$,

The coordinates of its center $C$ are $(0,1)$, the radius is $\sqrt{5}$, the distance from point $C(0,1)$ to the line $l$ is:

$$
d=\frac{|3 \times 0+1-6|}{\sqrt{3^{2}+1^{2}}}=\frac{5}{\sqrt{10}}<\sqrt{5}
$$

so the straight line $l$ intersects the circle $C$ and has two intersection points.

This example is not only the application of the new knowledge "The Position Relation between the Straight line and the Circle", but also the demonstration of this type of problem solving. What's more, the mathematics thought of "Combination of Number and Shape" and the mathematics methods "Algebra Method" (Solution method 1) as well as "Geometric Method" (Solution method 2) to solve the problems of conic curve are contained in this example. 


\subsubsection{Digging Deeply the Educational and Teaching Value of Exercises}

The exercises in mathematics textbooks are carefully designed and selected by the editors according to the knowledge of mathematics textbooks and the requirements of mathematics curriculum standards. They are of basic, typical, hierarchical and developmental characteristics and play an important role in consolidating and strengthening new knowledge, expanding and extending new knowledge and comprehensively applying new knowledge and so on [5]. However, there is a phenomenon of low utilization rate of exercises in mathematics textbooks. Many Students think that mathematics textbook exercises are "too few, too simple", question types are "single". What is worse, some students even think mathematics textbooks are "useless", thus they never read mathematics textbooks. [5] Instead, they turn to a large number of exercise books. This is not only a great waste of mathematics textbook resources, but also makes a greatly negative impact on the quality of education and teaching.

\subsubsection{To Deeply Explore the Educational and Teaching Value of the Expanding Curriculum Resources}

The expanding curriculum resources of columns such as "Reading and Thinking", "Exploration and Discovery" and "Mathematics in Life" also have unique educational and teaching value, which plays an important role in cultivating learning interest, broadening vision and enriching knowledge, etc. Mathematics teachers should attach great importance to them. For example, students can deepen their understanding of negative numbers, enjoy the glory of ancient Chinese mathematics history, and enhance their sense of national cultural identity by learning "China is the first country to use negative numbers" in the "Reading and Thinking" column of the Junior High School Mathematics Textbooks of Hunan Education Press. Students' inquiry ability can be cultivated and their innovative consciousness and practical ability can be improved by operating the tangram experiment in the column of "Inquiry and Discovery". Students can feel the omnipresence of mathematical culture in life and experience the beauty of mathematics by discussing the "Wonderful Golden Section" in the column "Mathematics in Life".

\subsection{Holding Mathematics Textbooks as a Whole}

The mathematics textbooks of primary and secondary schools select the core and basic knowledge of mathematics, and a structural system was arranged according to the logical order of mathematics, the rules of education and the law of physical and mental development of students, which is an organic whole and has a strong system. In order to deeply understand mathematics textbooks and use them scientifically and effectively, mathematics teachers need to grasp the mathematics textbooks as a whole, such as grasping the writing characteristics of the mathematics textbooks, the layout system, the basic structure, the presentation methods and so on. For example, the current junior high school mathematics textbooks are written in four sections "Number and Algebra", "Graphics and 
Geometry", "Statistics and Probability" and "Comprehensive Practice" [6]. Each section is relatively independent and related to each other. Therefore, mathematics teachers need to study the whole section, the whole chapter and even the complete set of mathematics textbooks.

\subsection{Using Mathematics Textbooks Creatively}

Paying attention to mathematics textbooks does not mean sticking to textbooks. The "Compulsory Education Mathematics Curriculum Standards (2011 Version)" clearly states that in teaching activities, mathematics teachers have to use materials in mathematics textbooks creatively in addition to using teaching materials in mathematics textbooks reasonably and effectively [6]. Mathematics textbooks are special texts written based on the general situation of our country, so it is impossible for mathematics textbooks to include all the teaching content and fully consider the actual situation of every school, every class, every teacher and every student. Therefore, in order to give full play to the educational and teaching value of mathematics textbooks, it is necessary to use mathematics textbooks creatively. We can do as follows: creating teaching situation flexibly according to the actual teaching situation, arranging class hours rationally, adapting examples and exercises in mathematics textbooks, adding or deleting mathematics textbooks content appropriately.

For example, the situation created by mathematics textbooks may not be suitable for teaching practice. Mathematics teachers can select teaching materials that are close to students' life apart from mathematics textbooks, and create new teaching situations. The Parallel Judgment of Line and Plane and its Properties of High School Mathematics Textbooks of Hunan Education Press is a good case. The content of which overemphasizes the logic derivation and axiomatic proof, and ignores the essence of life contained in mathematical objects. If mathematics teachers can select some of the life materials that are close to students, create vivid and interesting problem situations, such as the horizontal bar of the playground, the fan of the ceiling, the bridge of the city traffic, the fluorescent tube on the wall and so on, which can stimulate the students' interest in learning mathematics, reduce difficulty for students to understand new mathematics knowledge, make students feel that "Mathematics comes from life and serves life in return".

\subsection{Paying Attention to the Comparative Study and Use of Different Versions of Textbooks}

Since New Curriculum Reform, Chinese government has implemented a "One Standard and Multiple Copies" system in elementary education textbooks. As a result, the versions of mathematics textbooks for primary and secondary schools are diversified. Different versions of textbooks have their own characteristics, and there are differences in content selection, column setting, presentation mode, arrangement system and so on. Mathematics teachers can not only dee- 
pen their understanding of mathematics textbooks, but also get useful enlightenment from other versions of mathematics textbooks by a comparative study of different versions of mathematics textbooks. But how to conduct a comparative study of different versions of mathematics textbooks? Mathematics teachers can conduct a comparative study of mathematics textbooks from a horizontal and longitudinal perspective.

\subsubsection{Comparative Study from a Horizontal Perspective}

Comparative study from a horizontal perspective refers to the comparative study of mathematics textbooks published by different publishing houses. By comparing mathematics textbooks horizontally, we can discover the characteristics, advantages and disadvantages of mathematics textbooks published by different publishing houses, and learn from different mathematics textbooks to gain rich curriculum resources.

For example, by a comparative study of junior high school mathematics textbooks between the People's Education Press (PEP) and the Beijing Normal University Press (PNUP), we can find that there are differences between the two versions of mathematics textbooks in column setting, arrangement order and exercises and so on. In terms of column setting, the junior high school mathematics textbooks of PEP mainly have columns of "Thinking", "Exploration", "Practice", "Exercises", "Information Technology Application", "Reading and Thinking", etc. The junior high school mathematics textbooks of PNUP mainly have columns of "Doing", "Thinking", "Discussing", "Do Exercises in Class", "Exercises", "Reading" and so on. In terms of the arrangement order, although the mathematics knowledge in the two versions of mathematics textbooks is not much different, the arrangement order is quite different. For example, the "Triangle" of the junior high school mathematics textbooks of PEP is arranged in the first volume of eighth grade, while it is arranged in the second volume of seventh grade for PNUP.

\subsubsection{Comparative Study from a Longitudinal Perspective}

Comparative study from a longitudinal perspective refers to the comparative study of mathematics textbooks of different versions in the same publishing house. Through the longitudinal comparison of the new and old versions of mathematics textbooks, we can not only discover the changes and characteristics of the new mathematics textbooks, but also discover the changes in the concept of Mathematics Curriculum Standard, curriculum objectives, the nature of curriculum, the structure of curriculum, etc. By comparing different versions of junior high school mathematics textbooks of PEP, for example, it can be found that many new changes have taken place in the 2013 version than the 2004 version: In terms of knowledge presentation, the process of occurrence and development of mathematics knowledge has been paid more attention, which makes students experience the process of observing, doing experiment, guessing, reasoning, discussing, reflecting, etc. It aims to cultivate students' ability of inde- 
pendent thinking and independent inquiry, improve their sense of cooperation and realize the value of mathematics. In terms of course content, there is a significant increase in mathematics knowledge needed for social development, such as probability and statistics. In the exercises, it has been paid more attention to the use of mathematics knowledge to solve practical problems.

\section{Summary and Reflection}

The rapid development of information technology and the popularity of the Internet have brought a wealth of educational and teaching resources. Teachers can share or access high-quality educational and teaching resources anytime and anywhere on the Internet. And students can also get access to educational and teaching resources online as well as learn anytime and anywhere. However, this does not mean that the value of mathematics textbooks is weakened. On the contrary, the mathematics textbooks of primary and middle schools are still the basic elements of teaching, the main basis of teaching, the main materials of learning, the carrier of the functions of mathematics education, which plays an irreplaceable role in elementary education. If mathematics teachers pay attention to mathematics textbooks, understand the writing intentions of the mathematics textbooks, deeply excavate the educational and teaching value of mathematics textbooks, grasp the mathematics textbooks as a whole, use mathematics textbooks creatively, and pay attention to the comparative study and use of different versions of mathematics textbooks, it will give full play to the educational and teaching functions of mathematics textbooks.

\section{Acknowledgements}

This work is supported by the Key Projects of Undergraduate Teaching Reform Project of Higher Education in Guangxi in 2019 "Research and Practice of Effective Mathematics Teaching Oriented by Systematic Thinking Ability" (2019JGZ110) and Guangxi Zhuang Autonomous Region Postgraduate Education Innovation Project "Attribution Analysis of Mathematical Inverse Quotient of Junior Middle School Students in Minority Areas Based on NVIVO Coding" (XYCSZ2019084).

\section{Conflicts of Interest}

The authors declare no conflicts of interest regarding the publication of this paper.

\section{References}

[1] Yu, S.Q. and Wang, A.X. (2016) The Transformation Path for "Internet+ Education”. China Educational Technology, No. 10, 1-9.

[2] Gu, J. (2016) Investigation and Research into the Situation of Textbook Used by High School Mathematics Teachers. Suzhou University, Suzhou.

[3] Wu, L.B. and Qin, H. (2013) On the Functions of Examples in Middle School Mathematics Textbooks. Journal of Teaching and Management, No. 4, 53-55.

[4] Wu, L.B. and Wang, F.Y. (2014) Seven Functions of Exercises in Mathematics 
Textbooks. Journal of Teaching and Management, No. 31, 66-68.

[5] Yang, H.J. (2012) An Investigative Study on Use of New Mathematics Textbooks in Senior High School. Southwest University, Chongqing.

[6] Ministry of Education of the People's Republic of China (2011) Compulsory Education Mathematics Curriculum Standards (2011 Version). Beijing Normal University Press, Beijing. 